sexual health service in Birmingham, UK has provided free online home-based sampling in addition to a clinic-based service. The objectives of the study were to: - assess whether there were differences between the groups accessing screening online and in clinic; - evaluate the health outcomes associated with screening by setting; - analyse the interactions between online services and clinic-based services in terms of patient usage, and changes in access over time.

Methods A retrospective analysis of the clinic and online databases was undertaken to identify patients who undertook home-based and clinic-based testing between January and December 2017. Statistical analyses were undertaken to assess the uptake of screening by population group and identify predictors of screening uptake in different settings.

Results Overall 31,901 online testing kits were requested, with 18,087 returned, which equated to 14,667 patients. In the same period, 44,047 appointments were conducted in clinic, for 36,209 patients. A higher proportion of patients accessing online screening compared to clinic-based services were female $(66.3 \%$ vs $52.1 \%, \mathrm{p}<0.001)$, aged $<25$ $(52.5 \%$ vs $41.5 \%, \mathrm{p}<0.001)$, white $(74.4 \%$ vs. $40.1 \%$, $\mathrm{p}<0.001)$, asymptomatic $(79.6 \%$ vs. $49.4 \%, \mathrm{p}<0.001)$, and a lower proportion were from the two most deprived socio-economic groups $(38.8 \%$ vs. $50.5 \%, \mathrm{p}<0.001)$. There were also differences in positivity rates for chlamydia and gonorrhoea $(7.25 \%$ vs. $9.98 \%$ and $1.53 \%$ vs $3.47 \%$, $\mathrm{p}<0.001)$.

Conclusion This study provides valuable insights into differences in patient characteristics between those accessing online and clinic based services. This knowledge will allow those involved in planning and delivering services to understand how different service elements can complement each other. Our findings can be used to ensure that digital health services are integrated effectively alongside other types of services, in the context of limited resources, both in the UK and internationally.

Disclosure No significant relationships.

\section{P090 UNDERSTANDING YOUNG PEOPLE'S PRIORITIES FOR SEXUALLY TRANSMITTED INFECTION (STI) SCREENING}

${ }^{1}$ Louise Jackson*, ${ }^{2}$ Hareth Al-Janabi, ${ }^{2}$ Tracy Roberts, ${ }^{3}$ Jonathan Ross. ${ }^{1}$ University of Birmingham, Health Economics Unit, Birmingham, UK; ${ }^{2}$ University of Birmingham, Health Economics Unit, Birmingham, UK; ${ }^{3}$ University Hospital NHS Foundation Trust, Birmingham, UK

\subsection{6/sextrans-2019-sti.285}

Background It is important that STI screening provision reflects the priorities of young people, as they bear the greatest burden of disease. Such provision has become possible in a wider range of settings but there are constraints due to budget pressures. The objectives of the study were: - To assess how young people prioritise different characteristics of STI screening; - To analyse whether there are differences across socio-demographic groups; - To predict participation rates for different service configurations.

Methods Eight qualitative focus groups were used to design a discrete choice experiment (DCE) to analyse the choices made by young people. DCEs are an attribute-based survey method which involve respondents making choices between hypothetical scenarios, comprising two or more alternatives. The DCE included the following service characteristics: waiting times for appointments, waiting times for results, type of consultation, staff attitude, type of screening test, STIs tested for, and setting. The DCE was administered to 2000 young people who were part of an online panel in the UK, with quotas set to ensure inclusion of minority ethnic groups.

Results Analyses indicated that all seven service characteristics investigated were statistically significant factors for participants. Feeling that staff were non-judgemental was the most important characteristic to young people. Being tested for all STIs, having a full consultation and getting results quickly were also characteristics identified as important. Further analyses revealed some heterogeneity in priorities by gender, ethnicity and age group.

Conclusion This study provides valuable insights into the service characteristics that are seen as the most important by young people. This knowledge will allow those involved in providing and designing services to understand the relative importance of different service characteristics. At a time when sexual health services are facing pressures, such findings can be used to inform service development to ensure that decision-making is informed by young people's priorities.

Disclosure No significant relationships.

\section{P091 ESTIMATING NEONATAL HERPES SIMPLEX VIRUS INFECTIONS USING CHAPMAN'S CAPTURE-RECAPTURE METHOD, FLORIDA, 2011-2017}

${ }^{1}$ James Matthias*, ${ }^{2}$ Sonya Du Bernard, ${ }^{3}$ Gayle Keller, ${ }^{4}$ Julia Schillinger, ${ }^{5}$ Thomas Peterman, ${ }^{3}$ Craig Wilson. 'Centers for Disease Control and Prevention, Division of STD Prevention, Tallahassee, USA; ${ }^{2}$ University of Florida, Gainesville, USA; ${ }^{3}$ Florida Department of Health, STD and Viral Hepatitis Section, Tallahassee, USA; ${ }^{4}$ Centers for Disease Control and Prevention, New York City, USA; ${ }^{5}$ Centers for Disease Control and Prevention, Division of STD Prevention, Atlanta, USA

\subsection{6/sextrans-2019-sti.286}

Background Neonatal infection with Herpes Simplex Virus (nHSV) is a reportable condition in Florida. Healthcare providers are required to report cases of nHSV, and clinical laboratories are required to report the results of tests in which HSV is detected. However, electronic laboratory reporting (ELR) to the Florida Department of Health is incomplete, and results are not captured in the repository used for case-based reporting of other nationally reported sexually transmitted infections. We estimated the incidence of laboratory-confirmed nHSV in Florida using provider-reported cases alone, ELR alone, both provider and ELR reports, and the incidence yielded by a capture-recapture methodology.

Methods Provider-reported cases of nHSV (infants $\leq 60$ days of age with HSV infection confirmed by culture or polymerase chain reaction) during 2011-2017, and laboratory reports of HSV-positive culture or PCR results in the same age group, over the same period, were extracted and analyzed. Provider-reported cases were matched with ELR results using name, date of birth, and specimen collection dates. Chapman's estimator for capturerecapture was used to estimate nHSV incidence in Florida. Rates of nHSV infections per 100,000 live births were calculated.

Results Providers reported 113 nHSV cases and ELR identified 197 nHSV cases during 2011-2017. Of these, 44 cases were common to both datasets, leaving 266 unique nHSV reports. Given the number of unmatched cases, Chapman's estimator suggests 501 (95\% C.I. 401-600) nHSV cases occurred in Florida during the study period. The incidence of nHSV using 
only provider-reports was 7.4 cases/100,000 live births; ELRonly was 12.8 ; combined provider and ELR reports was 17.3; and Chapman's estimator was 32.7 .

Conclusion The incidence of nHSV measured using current provider- and ELR-reporting may substantially underestimate the disease burden in Florida. Expanding the number of healthcare facilities and laboratories electronically reporting nHSV infections and encouraging providers to report nHSV cases could help address the gap in reporting.

Disclosure No significant relationships.

\section{\begin{tabular}{|l|l}
\hline P092 HERPES SIMPLEX VIRUS TYPE 1 EPIDEMIOLOGY IN \\
\hline
\end{tabular} LATIN AMERICA AND THE CARIBBEAN: SYSTEMATIC REVIEW AND META-ANALYTICS}

'Layan Sukik, ${ }^{1}$ Mariam Alyafei, ${ }^{2}$ Manale Harfouche*, ${ }^{3}$ Laith Abu-Raddad. 'Q Qatar University, Department of Health Sciences, Doha, Qatar; ${ }^{2}$ Weill Cornell Medicine-Qatar, Infectious Disease Epidemiology Group, Doha, Qatar, ${ }^{3}$ Weill Cornell Medicine-Qatar, Doha, Qatar

\subsection{6/sextrans-2019-sti.287}

Background We aim to investigate the epidemiology of herpes simplex virus type 1 (HSV-1) in Latin America and the Caribbean.

Methods Systematic review and meta-analytics guided by the Cochrane Collaboration Handbook and reported following the PRISMA guidelines.

Results Thirty-one relevant reports were identified including 35 overall (and 95 stratified) seroprevalence measures, and five and seven proportions of virus isolation in genital ulcer disease (GUD) and in genital herpes, respectively. Pooled mean seroprevalence was $57.2 \%$ (95\% CI: 49.7-64.6\%) among children and 88.4\% (95\% CI: 85.2-91.2\%) among adults. Pooled mean seroprevalence was lowest at $49.7 \%$ (95\% CI: $42.8-$ $56.6 \%)$ in those aged $\leq 10$, followed by $77.8 \%$ (95\% CI: $67.9-84.8 \%$ ) in those aged 10-20, 82.8\% (95\% CI: 73.1$90.8 \%$ ) in those aged 20-30, 92.5\% (95\% CI: 89.4-95.1\%) in those aged 30-40, and 94.2\% (95\% CI: 92.7-95.5\%) in those aged $\geq 40$. Age was the strongest source of heterogeneity in seroprevalence, explaining $54 \%$ of variation. Evidence was found for seroprevalence decline over time. Pooled mean proportion of HSV-1 isolation was $0.9 \%$ (95\% CI: $0.0-3.6 \%$ ) in GUD and 11.1\% (95\% CI: 3.1-22.3\%) in genital herpes. Conclusion HSV-1 is a widely prevalent infection in this region, but its epidemiology appears to be slowly transitioning, with still limited contribution for HSV-1 in genital herpes.

Disclosure No significant relationships.

\section{P093 PERFORMANCE OF FOUR DIAGNOSTIC ASSAYS FOR DETECTING HERPES SIMPLEX VIRUS TYPE 2 ANTIBODIES IN MIDDLE EAST AND NORTH AFRICA}

${ }^{1}$ Soha Dargham*, ${ }^{2}$ Gheyath Nasrallah, ${ }^{2}$ Afifah Sahara, ${ }^{2}$ Malaz Elsidiq, 'Laith Abu-Raddad. ${ }^{1}$ Weill Cornell Medicine-Qatar, Doha, Qatar, ${ }^{2}$ Qatar University, Doha, Qatar

10.1136/sextrans-2019-sti.288

Background Assessments of commercial assays in detecting herpes simplex virus type 2 (HSV-2) antibodies have shown variable sensitivity and specificity, and variation in performance by global population. We aimed to evaluate performance of four assays in detecting HSV-2 antibodies in a composite Middle Eastern and North African (MENA) population. The assays are two ELISA kits: HerpeSelect 2 ELISA IgG and
Euroimmun Anti-HSV-2 (gG2) ELISA (IgG), and two immunoblot (IB)/Western blot (WB) assays: HerpeSelect 1 and 2 Immunoblot IgG and Euroimmun Anti-HSV-1/HSV-2 gG2 Euroline-WB (IgG/IgM).

Methods Blood specimens were drawn from blood donors between 2013-2016 in Doha, Qatar. Twenty specimens from ten nationalities (Egypt, Iran, Jordan, Lebanon, Pakistan, Palestine, Qatar, Sudan, Syria, and Yemen; total=200) were randomly selected and tested for HSV-2 antibodies.

Results In the six possible assay comparisons, Cohen's kappa statistics indicated fair to good agreement, ranging between 0.57 (95\% CI $0.28-0.86)$ and 0.69 (95\% CI $0.44-0.95)$. Meanwhile, positive percent agreement ranged between 50.0 (95\% CI 18.7-81.3\%) and 63.6\% (95\% CI 30.8-89.1\%); negative percent agreement ranged between $97.8 \% \quad(95 \% \mathrm{CI}$ 94.4-99.4\%) and 99.5\% (95\% CI 97.0-100.0\%); and overall percent agreement ranged between $95.8 \%$ (95\% CI 91.9 97.9\%) and $97.5 \%$ (95\% CI 94.2-98.9\%). The two ELISA kits demonstrated comparable sensitivities and specificities $\geq 50 \%$ and $>98 \%$, respectively, with respect to the IB/WB assays.

Conclusion The study provided, for the first time, primary data on performance of these assays in diagnosing HSV-2 infection in MENA populations. Findings support comparable performance and utility of these assays, and demonstrate challenges in establishing seropositivity (versus seronegativity).

Disclosure No significant relationships.

\section{P094 AN ASSESSMENT OF RISK FACTORS FOR HSV-2 INFECTION IN MALAWIAN WOMEN USING TWO CLASSIFICATIONS FOR THE HERPESELECT 2 TEST}

${ }^{1}$ Payal Chakraborty, ${ }^{1}$ Alison Norris, ${ }^{2}$ Sarah Huber-Krum, ${ }^{3}$ Sarah Garver, ${ }^{1}$ Robert Hood, ${ }^{4}$ Venson Banda, ${ }^{5}$ Abigail Norris Turner. ${ }^{1}$ The Ohio State University, College of Public Health, Division of Epidemiology, Columbus, USA; ${ }^{2}$ Harvard T.H. Chan School of Public Health, Department of Global Health and Population, Boston, USA; ${ }^{3}$ University of Chicago, Department of Sociology, Chicago, USA; ${ }^{4}$ Child Legacy International, Lilongwe, Malawi; ${ }^{5}$ Ohio State University, Internal Medicine, Infectious Diseases, Columbus, USA

10.1136/sextrans-2019-sti.289

Background The Focus HerpeSelect 2 ELISA IgG Test, used to diagnose herpes simplex virus type 2 (HSV-2) infection, is inexpensive, convenient, and widely used. However, past studies document poor specificity of this test in African populations. Increasing the index value cutpoint for a positive result improves specificity, but no studies to our knowledge have examined whether the correlates of HSV-2 infection change when the cutpoint for positivity changes. We investigated whether associations between select demographic and sexual risk factors and HSV-2 serostatus varied when the cutpoint for positivity was increased.

Methods We sampled women $(\mathrm{n}=218)$ from the Umoyo wa Thanzi project, an ongoing community-based cohort study in rural Malawi. Using multinomial logistic regression and accounting for village-level clustering, we examined unadjusted and adjusted associations between select risk factors and HSV2 serostatus. HSV-2 serostatus was coded in two ways: the manufacturer's recommended cutpoints $(<0.9=$ negative, $0.9-$ $1.1=$ indeterminate, $>1.1=$ positive), and modified cutpoints $(<0.9=$ negative, $0.9-3.5=$ indeterminate,$>3.5=$ positive $)$.

Results We assessed associations between HSV-2 serostatus and age, bacterial vaginosis (BV), and partner concurrency under 